Our trainees' day is also an introduction to the Trainees' Committee.

\section{Practical considerations}

In organising such a day, it is essential to liaise with the local postgraduate advisers and hospital clinical tutors who can encourage their new trainees to attend. Details of it should be circulated well in advance (3-4 weeks before the day) and a reminder is useful in the preceding week. The timing is important and the day should not clash with hospital induction courses. We have found that the second week of an attachment is most suitable.

Choice of venue affects attendance. It must be accessible both to attenders and speakers and should be booked in advance. Venues can often be arranged free of charge. Speakers and topics should be chosen using feedback from previous presentations. It is important that the speakers are of high quality, have been briefed to avoid overlap and are well prepared.

We have found that by addressing such minor practical difficulties it is possible to organise an induction day which is well accepted locally and is valued by those who attend. We believe there is scope to establish similar trainee led induction days elsewhere and urge other trainees' groups to do so.

\section{Reference}

Sims, A. C. P. (1990) The approval of psychiatric training schemes in 1988. Psychiatric Bulletin, 14, 338-342.

\title{
From pulsating brains to paranoid psychoses
}

\author{
Gillian Doody, Registrar in Psychiatry, Lynebank Hospital, Halbeath Road, \\ Dunfermline, Fife, Scotland KY11 4VW
}

The recent technological explosion in the advancement of brain imaging, resulting in MRI, SPECT and PET scanning, has served to further blur the interfaces of neurology, neurosurgery and psychiatry. As proposed by Reynolds (1990), the traditional divergence of classical neurological science and psychiatry, originating from Cullen's description of 'neurosis', is currently changing to convergence and in many areas coalescing.

Inspired by an overwhelming sense of neurological inadequacy, consequent upon entering psychiatry immediately on completion of my pre-registration year in 1988, I decided to equip myself with a basic level of neurological competence in an attempt to function optimally as a psychiatrist in the years ahead.

Having negotiated a six month 'leave of absence' from my registrar rotation in Fife, I then set about scouring the job advertisements in the British Medical Journal. Initially I had intended applying for neurology SHO posts, but was disheartened by job descriptions indicating that on call "acute medical knowledge and experience would be required". Inexplicably, I then gravitated to the neurosurgical columns and finally applied for a post as SHO in a large teaching neurosciences centre. Once appointed it was with a sense of great trepidation that I embarked on what was to be a memorable and emotive six months.

To one accustomed to district general psychiatry, the intricacies of a tertiary referral centre are intrinsically alien. The transition from dealing primarily with GP-style ailments to being in the forefront of state of the art medicine was initially a terrifying experience for the yokel psychiatrist. Being previously limited to inserting an intravenous cannula perhaps twice a year, the ability to perform umpteen daily arterial blood gases, lumbar punctures and place central venous lines with any degree of competence initially seemed an impossible dream. However, with multiple failures, frequent embarrassment and considerable patience from sympathetic registrars, experience tentatively emerged from a trial and error protocol.

The first four months of the attachment were spent fulfilling ward duties with no obligation to attend theatre or out-patient clinics. Routine admissions were plentiful for elective vascular procedures, tumour resection, shunt insertion, investigative cerebral angiography and myelography. In addition an average of four emergency admissions each day ranging from head injuries to acute spinal cord compression could be expected. 
Curiosity rapidly lured me to theatre, where I stood mesmerised when a craniotomy flap was lifted and dura parted to reveal pulsating living cortex. Gradually the incomprehensible two dimensional neuroanatomy textbook, with which I had struggled as a medical student and MRC Psych Part I candidate, transformed itself magically into an aide mémoire.

Of particular interest was the diverse spectrum of presentation of intracranial tumours from crude visual hallucinations in occipital lesions to short term memory impairment, seizures and non-specific personality change. In cases of aggressively malignant tumours, I was shocked at the rapid onset of symptomatology and often great physical integrity, despite complete lack of insight, which I observed in those with such a desperately poor prognosis. Occasionally such denial could be attributed to cognitive or perceptual disturbance consequent upon the primary pathology. Although this often served to provide a health adaptation on the part of the patient, the converse effect of pathological maladaptation could be witnessed in relatives (Strauss $e t$ al, 1990). The tragic plights of families were frequently evident and Macmillian nurses played an invaluable supportive role in a field to which it seemed frustratingly little hospital time could be allocated.

Psychosocial issues also featured prominently in the wake of young traumatically brain damaged adults. Those who, following only a milliseconds injury, remain chronically disabled and exhibit a range of atypical psychopathology, demonstrated the necessity for prompt multidisciplinary team involvement (McClelland, 1988) within a rehabilitative setting. Progress, however, was often agonisingly slow and the memory of an anxious mother bringing chocolates and fruit to her intubated, tube fed and unresponsive son on a daily basis still lingers.

The final two months of the appointment were spent as SHO responsible for a nine bedded neurosurgical high dependency unit dealing only with the acutely ill. Fortunately by this stage the previously ever-present anxiety of encountering a medical emergency had been partially extinguished by repetitive flooding. A distinct atmosphere of expected professional detachment seemed to prevail among staff, producing temporary ostracisation and embarrassment if rules were broken and tears were shed, as I learnt to my cost. Often a dry and sarcastic wit was utilised to express personal and emotional responses in a socially acceptable format, a game which I found distressingly easy to play.

Surprisingly, the most stressful period in the high dependency unit was spent dealing with a peripheral psychiatric problem, which evolved in an exclusively French-speaking relative. An elderly lady flew from abroad following a threat from her son to jump from the roof of a psychiatric hospital. She arrived to find him ventilated and critically ill having sustained diffuse brain injury from the fall. For six days and nights she refused to leave the bedside, would not eat, drink, sleep, wash or visit the toilet, attacking nurses who tended to her son. A French-speaking priest translated her beliefs that the anaesthetic gases were poisoning her, that she was being kept hostage with her son, who was being drugged by me and who, in my capacity as devil's envoy, I was attempting to kill. Shortly after this revelation a sudden deterioration in the son's condition necessitated transient transfer to the neuroradiology department for an urgent CT scan. At this point mother assumed her son was being taken to the mortuary and absconded in a distraught state. Extensive searching involving embassy officials and police located her 24 hours later at Heathrow Airport from where she was escorted back home to be met by family.

Having now returned to Fife the task of assessing the experience gained in the last six months is perhaps best left to future years. However, the over-riding memory is not of the acquisition of a knowledge of neurological symptoms and signs, although this was certainly achieved. My lasting impression is of a privileged insight into people's personal tragedies, coping strategies and idiosyncrasies in the face of adversity.

\section{References}

MCClelland, R. J. (1988) Psychological sequelae of head injury-anatomy of a relationship. British Journal of Psychiatry, 153, 141-146.

REYNOLDS, E. H. (1990) Structure and function in neurology and psychiatry. British Journal of Psychiatry, 157, 481-490.

Strauss, D. H., Spitzer, R. L. \& Muskin, P. R. (1990) Maladaptive denial of physical illness: a proposal for DSM-IV. American Journal of Psychiatry, 147, $1168-1172$. 